\title{
Molecular Weight Dependence of Viscoelasticity of Polycaprolactone Critical Gels
}

\author{
Akihiro Izuka and H. Henning Winter* \\ Chemical Engineering Department, University of Massachusetts, \\ Amherst, Massachusetts 01003 \\ Takeji Hashimoto \\ Department of Polymer Chemistry, Faculty of Engineering, Kyoto University, \\ Kyoto 606, Japan \\ Received September 19, 1991; Revised Manuscript Received January 2, 1992
}

\begin{abstract}
The molecular weight dependence of critical gel properties was determined for poly( $\epsilon$-caprolactone) diol end-linked with a three-functional isocyanate. The critical gels exhibit the typical scaling behavior with a power law relaxation spectrum $H(\lambda)=G_{0} / \Gamma(n)\left(t / \lambda_{0}\right)^{-n}, \lambda>\lambda_{0}$, where $G_{0}=G_{\theta}$ was found to be the modulus of the fully cross-linked polymer and $\lambda_{0}=\eta_{0} / G_{e}$ was found to depend on the viscosity of the difunctional prepolymer, $\eta_{0}$. The relaxation exponent, $n$, decreases with increasing cross-linker concentration (increasing stoichiometric ratio, $r=[\mathrm{NCO}] /[\mathrm{OH}]$ ) and increasing molecular weight of the prepolymer. This suggests that the fractal dimension of the critical gel increases with increasing molecular weight and stoichiometric ratio.
\end{abstract}

\section{Introduction}

The mechanical properties of polymeric materials are highly dependent on molecular weight. The zero-shear viscosity $\left(\eta_{0}\right)$ of a large number of polymeric liquids was found to depend on the weight-average molecular weight $\left(M_{\mathrm{w}}\right)$ as

$$
\eta_{0} \propto M_{\mathrm{w}}^{\alpha}
$$

with $1 \leq \alpha \leq 2.5$ for $M_{\mathrm{w}} \leq M_{\mathrm{c}}$ and $\alpha=3.4$ for $M_{\mathrm{w}} \geq M_{\mathrm{c}}$ where $M_{\mathrm{c}}$ is the entanglement molecular weight. ${ }^{1,2}$ The equilibrium modulus $\left(G_{e}\right)$ of a rubbery solid decreases with the molecular weight between cross-linking points, $M_{\mathrm{e}}:^{3}$

$$
G_{\mathrm{e}} \propto 1 / M_{\mathrm{e}}
$$

Although these relationships are well understood for polymeric liquids and solids, little is known about the molecular weight dependence of the mechanical properties of a cross-linking polymer which undergoes a transition from a liquid to a rubbery solid. The average molecular weight increases and the molecular weight distribution broadens with increasing extent of reaction, and both diverge at the gel point. The molecular weight of endlinking polymers before cross-linking determines the strand lengths in the evolving network. It, therefore, is assumed that the molecular weight of the initial polymer components $\left(M_{\mathrm{n}}\right)$ is an important parameter for the gel behavior.

Recently, rheological experiments have revealed that the critical gel (CG), which is the ideal material exactly at the gel point (GP), exhibits a continuous power law relaxation time spectrum (CW spectrum $)^{4,5}$

$$
H(\lambda)=H_{0} \lambda^{-n}, \quad \lambda_{0}<\lambda<\infty
$$

which results in a relaxation modulus

$$
G(t)=\int_{0}^{\infty} H(\lambda) e^{-t / \lambda} \mathrm{d} \lambda / \lambda \cong S t^{-n}, \quad \lambda_{0}<t
$$

Gel strength $\left(S=H_{0} \Gamma(n)\right)$ and relaxation exponent $(n)$ are the only material parameters which characterize the linear viscoelastic properties. $\lambda_{0}$ indicates the shortest time of the power law behavior (lower cutoff) due to glass transition or entanglement effects. The same scaling behavior is also apparent in dynamic mechanical experiments where the storage modulus $\left(G^{\prime}\right)$ and the loss modulus $\left(G^{\prime \prime}\right)$ at GP are

$$
G^{\prime}=G^{\prime \prime} / \tan \delta=S \omega^{n} \Gamma(1-n) \cos \delta, \quad 0<\omega<1 / \lambda_{0}
$$

The phase angle $(\delta)$ between stress and strain is independent of frequency $(\omega)$ but proportional to the relaxation exponent

$$
\delta=n \pi / 2
$$

The initial experimental studies ${ }^{4-6}$ for the determination of $S$ and $n$ focused on a cross-linking poly(dimethylsiloxane) (PDMS) system, and the effects of stoichiometry and dilution have been investigated in great detail. The value of $n$ varied over about the entire range between 0 and 1, depending on molecular composition and crosslinking conditions.

Theories ${ }^{7-10}$ predict the power law behavior of CGs as well as the exponent $(n)$ in conjunction with a fractal dimension which derives from the statistical self-similarity. Some theories actually suggest that the exponent $(n)$ should be independent of molecular structure.

The complicated dimensions of $S, \mathrm{~Pa} \mathrm{~s}{ }^{n}$, suggest that it is composed of a material characteristic modulus and a time. ${ }^{4}$ Experiments can be modeled with a relation between $S$ and $n$ of the form ${ }^{6}$

$$
S=G_{0} \lambda_{0}{ }^{n}
$$

where $\lambda_{0}$ and $G_{0}$ are material-specific constants. It was found that $G_{0}$ and $G_{0} \lambda_{0}$ were very close to the modulus of the fully cross-linked material and the zero-shear viscosity of the prepolymer, respectively.

For this study, we selected an end-linking polyurethane system consisting of poly( $\epsilon$-caprolactone) diol (PCL) of various molecular weights and triisocyanate. PCL is usually synthesized by ring-opening polymerization of $\epsilon$ caprolactone. The molecular weight is controlled by adjusting the ratio of monomer to initiator. PCL is a technologically important material which is mainly used as a polyurethane resin in combination with other polyol components. The evolution of viscosity of PCL urethane networks during the cross-linking reaction has previously 
(a)

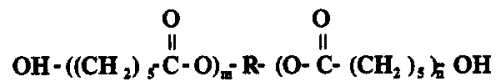

$$
\begin{aligned}
& \text { R: } \mathrm{CH}_{2}-\mathrm{CH}_{2}
\end{aligned}
$$

(b)

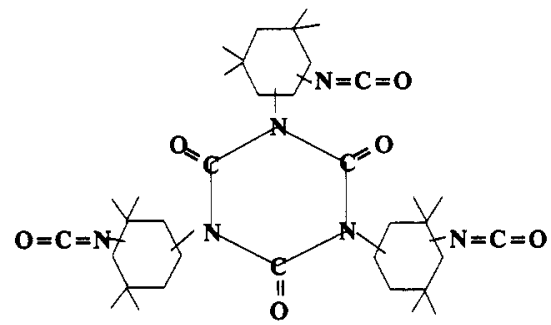

(a) Poly (E-caprolactone) diol (PCL)

(b) isocyanurate of isophorone diiscyanate (poly-IPDI)

Figure 1. (a) Poly (6-caprolactone) diol (PCL); (b) isocyanurate of isophorone diisocyanate (poly-IPDI).

Table I

Molecular Characterization of PCL

\begin{tabular}{llllll}
\hline & PCL2 & PCL4 & PCL7 & PCL9 & PCL20 \\
\hline$M_{\mathrm{n}}$ (titration) & $2.0 \times 10^{3}$ & $3.8 \times 10^{3}$ & $6.6 \times 10^{3}$ & $9.2 \times 10^{3}$ & $1.9 \times 10^{4}$ \\
functionality & 1.99 & 1.98 & 1.89 & 1.88 & 1.9 \\
$M_{\mathrm{w}} / M_{\mathrm{n}}$ & 2.18 & 2.15 & 2.12 & 2.10 & 2.08
\end{tabular}

been studied in terms of the kinetics. ${ }^{11,12}$ The effects of cross-linking on mechanical properties and crystallization behavior of PCL are also of interest. ${ }^{13,14}$

Here we investigate the effect of prepolymer molecular weight and stoichiometry on the characteristics of CGs, including the values of $n, G_{0}$, and $\lambda_{0}$.

\section{Experimental Conditions}

Materials and Their Characterization. Five poly( $\epsilon$-caprolactone) diols (PCLs) (commercial polymers of Daicel Chemical) were end-linked with a three-functional cross-linker, IPDI-T1890, supplied by Huels AG (poly-IPDI) (see Figure 1). The PCLs were dried at $100^{\circ} \mathrm{C}$ for $14 \mathrm{~h}$ under vacuum before use to remove traces of low molecular weight derivatives as well as water. Because $\mathrm{COOH}$ is generated when water initiates the ring-opening polymerization of $\epsilon$-caprolactone and the ester bond of PCL is hydrolyzed, the end groups of the PCL should be $\mathrm{OH}$ and $\mathrm{COOH}$. The two groups were quantitatively analyzed by titration, and number-average molecular weight and functionality were calculated based on these values. All PCLs in this study are polydisperse, with $M_{w} / M_{n}$ of approximately 2 as determined by GPC. These characteristics are summarized in Table I.

The poly-IPDI is virtually an isocyanurate of isophorone diisocyanate containing three free NCO groups per molecule (see Figure 1). The manufacturer's information specifies the NCO content in the compound as $17.5 \%$ by weight, although this value is calculated as $18.3 \%$ for the trimer. The poly-IPDI is a solid with a melting point of $110^{\circ} \mathrm{C}$. It was used without further purification.

Stoichiometrically balanced mixtures of PCL/poly-IPDI were prepared for each PCL. In addition, stoichiometrically imbalanced mixtures were prepared for PCL7, which is the best candidate for studying stoichiometric effects because its critical gels show measurable dynamic moduli over a wide range of stoichiometric ratio. The stoichiometric ratio $(r)$ of the system is defined here as the initial molar ratio of isocyanate group to hydroxy group, [NCO]/[OH]. From Flory and Stockmayer's theory of gelation ${ }^{15}$ and assuming that the functionalities of PCL and poly-IPDI are exactly equal to 2 and 3 , respectively, this system is expected to form critical gels for stoichiometric ratios in the range $0.5 \leq r \leq 2$. For covering this range, mixtures with $r=0.61,0.80,1.00,1.12,1.20,1.41$, and 1.76 were prepared.

For calculation of composition, we used an NCO content in the poly-IPDI of $17.5 \%$ by weight on the $\mathrm{OH}$ value of each PCL determined by the titration. A 60 wt \% solution of the polyIPDI in dried dichloromethane was added to the melted PCL

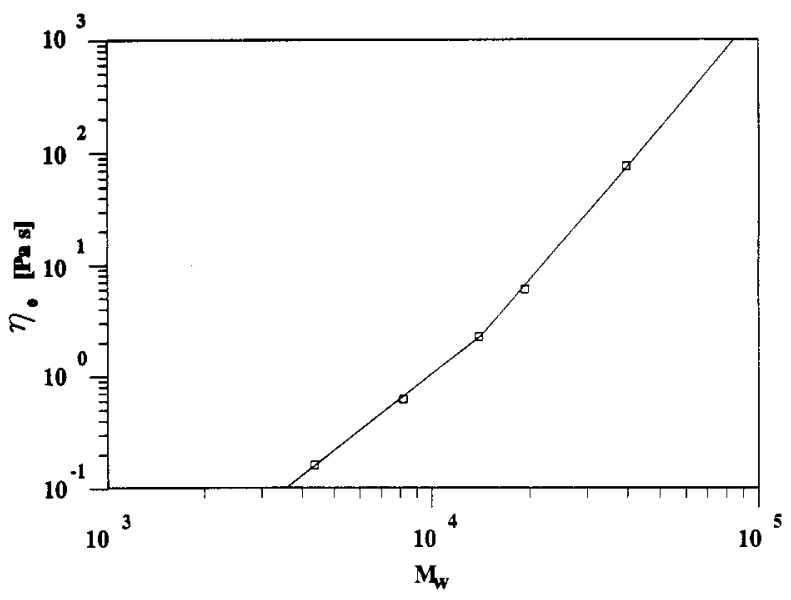

Figure 2. Zero-shear viscosities $\eta_{0}$ of PCLs at $110^{\circ} \mathrm{C}$ plotted against weight-average molecular weight $M_{\mathrm{w}}$. The $\eta_{0}$ was measured as the complex dynamic viscosity with the frequency range between 0.1 and $10 \mathrm{rad} / \mathrm{s}$.

and stirred thoroughly at $80^{\circ} \mathrm{C}$ for $5 \mathrm{~min}$ to get a homogeneous mixture. The mixture was then dried at $100^{\circ} \mathrm{C}$ under vacuum for $10 \mathrm{~min}$ to remove the solvent, cooled to room temperature, and transferred into the rheometer.

Rheological Experiments. Oscillatory shear measurements in a Rheometrics dynamic mechanical spectrometer used parallel plates at a maximum strain amplitude of $10 \%$. All samples were reacted isothermally at $110^{\circ} \mathrm{C}$ under a dried air atmosphere. This temperature is high enough above the melting point of PCL and, judging from the transparency of the samples, neither phase separation between the two components nor crystallization of the cross-linker occurred. The evolution of the dynamic modulus during cross-linking was measured over a span of two decades of frequency by consecutive frequency scans. Each scan took less than $2 \mathrm{~min}$. Interpolation of the mechanical properties was used to determine the state at which $\tan \delta$ is constant over the entire frequency range.

DSC Measurements. DSC experiments were performed under a helium atmosphere in a Perkin-Elmer DSC-2C with a heating rate of $20 \mathrm{~K} / \mathrm{min}$ from 150 to $280 \mathrm{~K}$. The glass transition temperature $\left(T_{\mathrm{g}}\right)$ reported here is a midpoint of the secondary transition in the DSC trace.

Prepolymer Properties. The complex dynamic viscosity $\left(\eta^{*}\right)$ of all the prepolymers at $110^{\circ} \mathrm{C}$ was independent of frequency between 0.1 and $10 \mathrm{rad} / \mathrm{s}$; i.e., all PCLs behaved as Newtonian fluids and $\eta^{*}$ may be denoted by the zero-shear viscosity, $\eta_{0}$. In Figure 2, these values are plotted against the weight-average molecular weight. The viscosities of the PCLs with small molecular weights (PCL2, PCL4, and PCL7) appear on a straight line with a slope of 2.15. The others (PCL9 and PCL 20) are above this line and seem to be on a line with a slope of 3.4. The molecular weight corresponding to the intersection of the two lines, which implies the entanglement molecular weight $\left(M_{\mathrm{c}}\right)$, is slightly larger than the molecular weight of PCL7. The lowest value of $T_{\mathrm{g}}$ was found in PCL2. However, the $T_{\mathrm{g}}$ of each prepolymer was in a very narrow range between 206 and $209 \mathrm{~K}$.

\section{Results on Stoichiometrically Balanced Systems}

Properties on Fully Cross-Linked Samples $(r=1)$. Fully cross-linked samples provide useful information which helps to understand the CG properties. Stable samples with balanced stoichiometry were prepared from the same mixtures as were used for the determination of $\mathrm{GP}$ by curing in the rheometer at $130^{\circ} \mathrm{C}$. After $14 \mathrm{~h}$ of curing we confirmed that the dynamic moduli did not change anymore and that the cross-linking reaction was brought to the highest possible conversion $(p \rightarrow 1)$. Then mechanical dynamic experiments were performed at 110 ${ }^{\circ} \mathrm{C}$ and at frequencies between 0.1 and $100 \mathrm{rad} / \mathrm{s}$. The modulus $\left(G^{*}\right)$ of all fully cross-linked PCL samples, except for PCL20, was almost independent of frequency as shown 


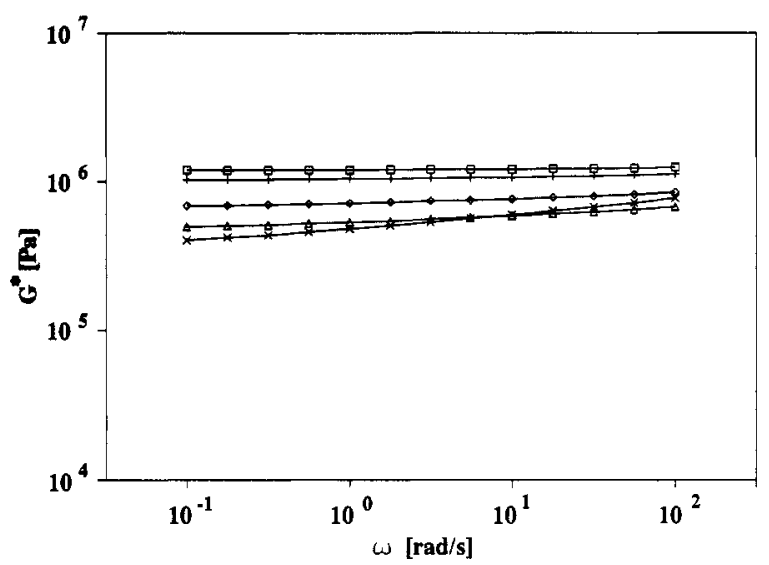

Figure 3. Complex dynamic moduli $G^{*}$ of the fully cross-linked materials of PCL2 (D), PCL4 (+), PCL7 $(\diamond)$, PCL10 $(\Delta)$, and PCL20 $(X)$ measured at $110^{\circ} \mathrm{C}$ with shear strain of $1 \%$ plotted against frequency $\omega$. As the dynamic loss moduli $G^{\prime \prime}$ were more than 1 order less than the dynamic storage moduli $G^{\prime}, G^{\prime}$ were almost equal to $G^{*}$.

in Figure 3. Its value was assumed to be equal to the equilibrium modulus $\left(G_{e}\right)$. $G^{*}$ for PCL20 depends on frequency and $G_{\mathrm{e}}$ could not be determined conclusively. However, its value must be very close to $G^{*}$ at low frequencies. Here we adopted the value of $G^{*}$ measured at $\omega=0.1 \mathrm{rad} / \mathrm{s}$ as $G_{e}$ for further calculations. As eq 2 suggests, $G_{\mathrm{e}}$ decreased with increasing molecular weight of the PCL which determines the molecular weight between cross-linking points, $M_{\mathrm{e}}$.

The molecular weight dependence of $T_{\mathrm{g}}$ for the fully cross-linked samples was somewhat larger than in the prepolymer (see Table II). Even then, the difference between $T_{\mathrm{g}}$ of PCL2 and PCL20 was still only $8 \mathrm{~K}$. Vitrification had no influence on the following experiments.

Critical Gels $(r=1)$. The $\tan \delta$ decreases with reaction time as shown in Figure 4 for PCL7 with the frequency as a parameter. We do not know the extent of reaction $(p(t))$ and simply plot the measured $\tan \delta(\omega)$ as a function of time distance from the gel point, $\left(t-t_{c}\right)$. The GP, which is easily detected by its self-similarity $(\delta=$ constant over the terminal frequency range), occurred for PCL7 at about $49 \mathrm{~min}\left(t_{c}\right)$ after the measurement had started. This time is experimentally important even if it cannot be interpreted because the cross-linking reaction has already proceeded during the sample preparation. For other PCLs, similar results were obtained and GPs could be clearly determined. $G^{\prime}$ and $G^{\prime \prime}$ of each PCL at GP exhibited a power law behavior over the entire observed frequency range (see Figure 5). $S$ and $n$ according to eqs 5 and 6 are summarized in Table III.

The relaxation exponent was in the range $0.31 \leq n \leq$ 0.91 ; i.e., it covered almost the entire possible range $(0<$ $n<1$ ). A low $n$ value implies that the material is a mostly elastic body with the limit of $G^{\prime \prime}=0$ at $n=0$ and vice versa.

In Figure 6 the relaxation exponent and the gel strength are plotted against the number-average prepolymer molecular weight, $M_{\mathrm{n}}$. The exponent decreases with increase of $M_{\mathrm{n}}$ while $S$ increases with increase of $M_{\mathrm{n}}$. This result offers a remarkable contrast with the mechanical properties of the fully cross-linked material in which $G_{\mathrm{e}}$ decreases with increase of $M_{n}$.

\section{Results on Stoichiometrically Imbalanced Systems}

Properties of Fully Cross-Linked Materials ( $r \neq$ 1). Fully cross-linked samples of stoichiometrically im- balanced systems were prepared through the same procedure as before, and dynamic experiments were performed at the same temperature and frequencies. The modulus $\left(G^{*}\right)$ of the fully cross-linked samples was almost independent of frequency as shown in Figure 7. The only real exception is the sample with $r=0.61$.

The values of $G^{*}$ at $\omega=0.1 \mathrm{rad} / \mathrm{s}$ are assumed to be equal to $G_{e}$. In Figure 8, they are plotted against $r$. The maximum value of $G^{*}$ should be theoretically observed in the stoichiometrically balanced system, which has no loose strands left in the network structure. In a real end-linking system, however, the cross-linking reaction cannot complete perfectly due to the poor mobility of partially reacted cross-linkers at the late stage of the reaction. We should expect the largest values of $G^{*}$ at $r$ slightly larger than 1 . The maximum was observed at $r=1.12$. A computer simulation of end-linking systems ${ }^{16}$ supports the trend that cross-linker-deficient mixtures produce less effective elastic networks than those using excess cross-linker. The samples' $G^{*}$ values were distributed over more than one decade, from $9.47 \times 10^{4}(r=0.61)$ to $1.50 \times 10^{6}(r=1.12)$.

Although the $T_{\mathrm{g}}$ 's of these materials have not been determined, the increase of $T_{\mathrm{g}}$ induced by cross-linking is expected to be less than that of the stoichiometrically balanced system due to the imperfection of network structure.

Critical Gels $(r \neq 1)$. Again, $\tan \delta$ decreases with reaction time as exemplified in Figure 9 for the system with $r=1.76$. The GP occurred at about $107 \mathrm{~min}\left(t_{\mathrm{c}}\right)$ after the measurement had started. For other stoichiometrically imbalanced systems, similar results were obtained and GPs could be clearly determined. The power law behavior in the dynamic moduli is demonstrated in Figure 10. $S$ and $n$ are summarized in Table IV. The relaxation exponent was in the range $0.34 \leq n \leq 0.67$; i.e., it covered the middle part of the possible range.

In Figure 11 the relaxation exponent and the gel strength are plotted against the stoichiometric ratio, $r$. The exponent monotonically decreases with increase of $r$ while $S$ increases with increase of $r$.

\section{Discussion}

Magnitude of Molecular Weight Dependent $S=$ $G_{0} \lambda_{0}{ }^{n}$. The upper and lower bounds of $S$ are given by the dynamic modulus of the fully cross-linked material and the viscosity of the un-cross-linked material, respectively. The dimension of $S$ coincides with that of the modulus and viscosity at the respective limits of $n \rightarrow 0$ and $n \rightarrow$ 1. As the viscosity and modulus depend on molecular weight, we would assume that $S$ also depends on molecular weight. Among our test samples, PCL2 has the lowest viscosity before cross-linking and the highest modulus when cross-linked completely. Therefore for all samples, $S$ must lie between $\sim 10^{-1} \mathrm{~Pa}$ and $\sim 10^{6} \mathrm{~Pa}$. The observed values of $S$ are distributed in the range $5.1 \times 10^{-1}\left(\mathrm{~Pa} \mathrm{~s}^{0.91}\right)$ $\leq S \leq 4.7 \times 10^{4}\left(\mathrm{~Pa} \mathrm{~s}^{0.31}\right)$, which covers almost all of the possible range.

$S$ and $n$ are strongly coupled as shown in Figure 12. One could attempt a straight line through the data according to eq 7 . A least-squares fit (not shown) would give $G_{0}=9.77 \times 10^{6} \mathrm{~Pa}$ and $\lambda_{0}=3.09 \times 10^{-8} \mathrm{~s}$ for the parameters in eq 7, and $G_{0} \lambda_{0}$ is calculated as $3.02 \times 10^{-1}$ $\mathrm{Pa}$ s. As Scanlan et al. ${ }^{6}$ found in the PDMS system, $G_{0}$ and $G_{0} \lambda_{0}$ are of the same order of magnitude as the equilibrium modulus of the fully cross-linked materials $\left(G_{e}\right)$ and the zero-shear viscosity of the prepolymer $\left(\eta_{0}\right)$, respectively. With the new data we can explore this relation further. If we assume that $G_{0}$ and $G_{0} \lambda_{0}$ perfectly 
Table II

Properties of Prepolymers and Fully Cross-Linked Materials $(r=1)$

\begin{tabular}{llllll}
\hline & \multicolumn{1}{c}{ PCL2 } & \multicolumn{1}{c}{ PCL4 } & \multicolumn{1}{c}{ PCL7 } & PCL9 & PCL20 \\
\hline$\eta_{0}, \mathrm{~Pa}$ s & $1.63 \times 10^{-1}$ & $6.30 \times 10^{-1}$ & 2.30 & 6.12 & $7.89 \times 10^{1}$ \\
$T_{\mathrm{g}}, \mathrm{K}$ (prepolymer) & 206 & 209 & 208 & 209 & 209 \\
$G_{\mathrm{e}},{ }^{a} \mathrm{~Pa}$ & $1.19 \times 10^{6}$ & $1.03 \times 10^{6}$ & $6.88 \times 10^{5}$ & $4.95 \times 10^{5}$ & $4.03 \times 10^{5}$ \\
$T_{\mathrm{g}}, \mathrm{K}$ (cross-linked material) & 222 & 216 & 214 & 214 & 214
\end{tabular}

${ }^{a}$ Measured as $G^{*}$ at $110^{\circ} \mathrm{C}$ at $\omega=0.1 \mathrm{rad} / \mathrm{s}$.

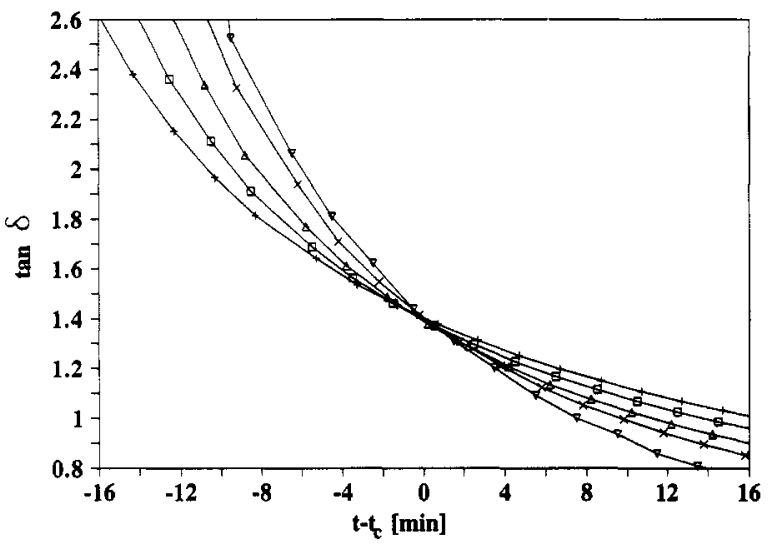

Figure 4. Evolution of $\tan \delta$ of PCL7 during isothermal crosslinking reaction at $110^{\circ} \mathrm{C}$ with the frequency of $1(\nabla), 3.162(X)$, $10(\Delta), 31.62(\square)$, and $100(+) \mathrm{rad} / \mathrm{s}$. The maximum strain amplitude was $10 \%$.

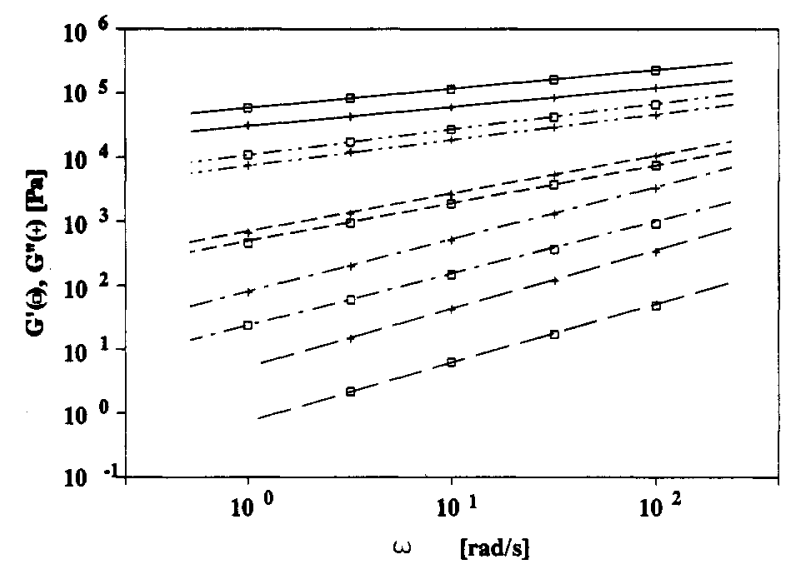

Figure 5. Dynamic storage $G^{\prime}(\square)$ and loss $G^{\prime \prime}(+)$ moduli for PCL20 (-), PCL10 (- - ), PCL7 (- - ), PCL4 (- - ), and PCL2 $(--)$ at the gel point plotted against frequency $\omega$. The temperature was $110^{\circ} \mathrm{C}$ and the maximum strain amplitude was $10 \%$.

Table III

Relaxation Exponent $n$ and Gel Strength S of PCL Critical Gels

\begin{tabular}{llllll}
\hline & PCL2 & PCL4 & PCL7 & PCL9 & PCL20 \\
\hline$n$ & 0.91 & 0.83 & 0.61 & 0.38 & 0.31 \\
$S, \mathrm{~Pa} \mathrm{~s}^{n}$ & $5.1 \times 10^{-1}$ & $1.5 \times 10^{1}$ & $3.6 \times 10^{2}$ & $9.5 \times 10^{3}$ & $4.7 \times 10^{4}$
\end{tabular}

coincide with $G_{e}$ and $\eta_{0}$, then $S$ and $n$ should be located on lines represented as

$$
S=G_{\mathrm{e}}\left(\eta_{0} / G_{\mathrm{e}}\right)^{n}
$$

where the two parameters are functions of molecular weight. In fact, we might be able to describe the molecular weight dependence of $S$ through the well-known molecular weight dependence of $G_{\mathrm{e}}$ and $\eta_{0}$; see eqs 1 and 2. For each of the samples, $G_{\mathrm{e}}$ and $\eta_{0}$ have been determined initially (Table II), and $n$ is known from $G^{\prime}$ and $G^{\prime \prime}$ measurements of the critical gel.

Figure 12 shows a comparison of the experimental $S$ values (squares) with the corresponding calculated values

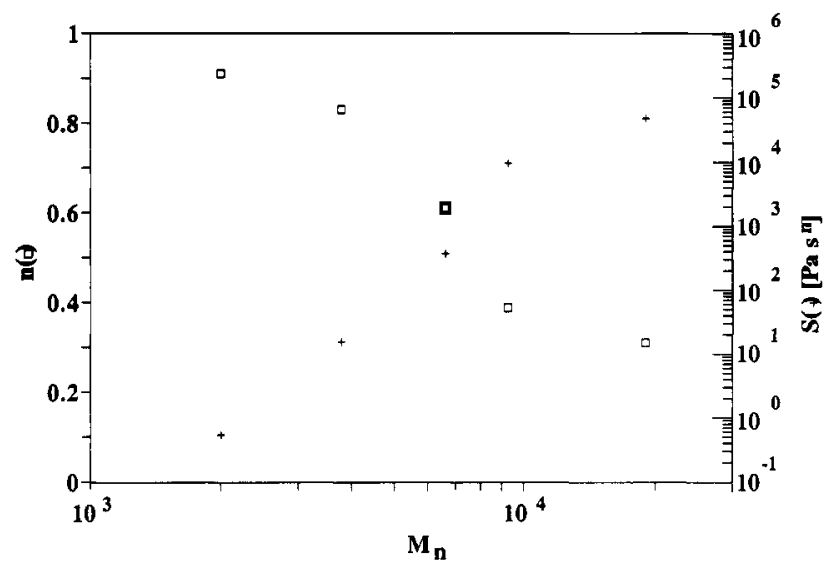

Figure 6. Relaxation exponent $n(\square)$ and gel strength $S(+)$ plotted against the number-average prepolymer molecular weight $M_{\mathrm{n}}$.

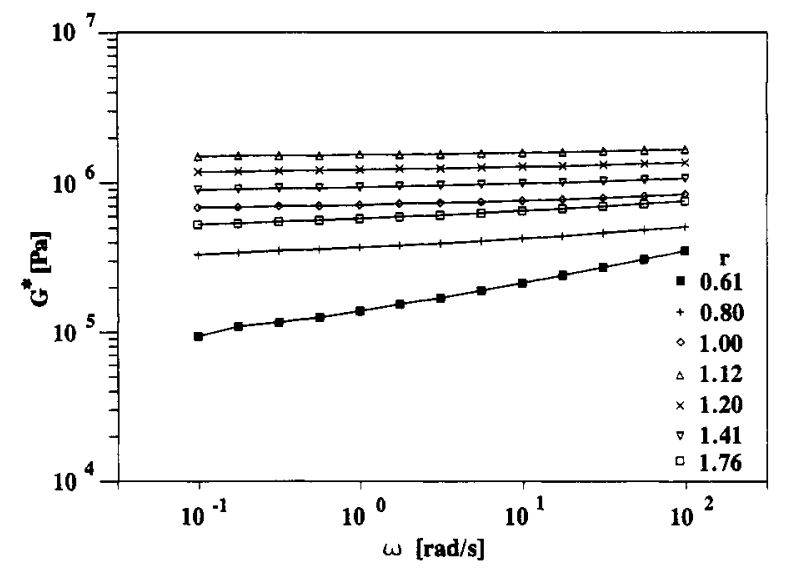

Figure 7. Complex dynamic moduli $G^{*}$ of the fully cross-linked materials of PCL7/poly-IPDI measured at $110^{\circ} \mathrm{C}$ with shear strain of $1 \%$ plotted against frequency $\omega$.

(crosses on dashed lines according to eq 8). There are only small discrepancies between calculated and experimental values, and the shapes of the curves are very similar. This observation may suggest that $G_{0}$ and $\lambda_{0}$ are functions of prepolymer molecular weight and that their values are close to $G_{e}$ and $\eta_{0} / G_{\mathrm{e}}$. A particular value of $n$ is observed for each of the gels depending on its prepolymer molecular weight, as long as $r=1$.

Stoichiometry Dependence of $S$. The values of $S$ and $n$ change vastly with composition, even for constant molecular weight of the prepolymer. PCL7 is used as an example and, according to the preceding discussion, its $S$ is expected to be in the range between $\sim 1 \mathrm{~Pa}$ s and $10^{6}$ $\mathrm{Pa}$. The observed values of $S$ are distributed in the range $8.8 \times 10^{1}\left(\mathrm{~Pa} \mathrm{~s}{ }^{0.67}\right) \leq S \leq 1.9 \times 10^{4}\left(\mathrm{~Pa} \mathrm{~s}^{0.34}\right)$.

The equilibrium modulus $\left(G_{e}\right)$ is one of the parameters in the model (eq 8). This equilibrium modulus strongly depends on stoichiometry. It has a maximum at about $r$ $\simeq 1$ and vanishes as $r$ approaches its upper and lower critical stoichiometric ratio, $r \rightarrow 2$ and $r \rightarrow 0.5$. For a first attempt at describing the data, we choose the maximum value $G_{\mathrm{e}, \max }$, found at $r=1.12$, as a reference value. 


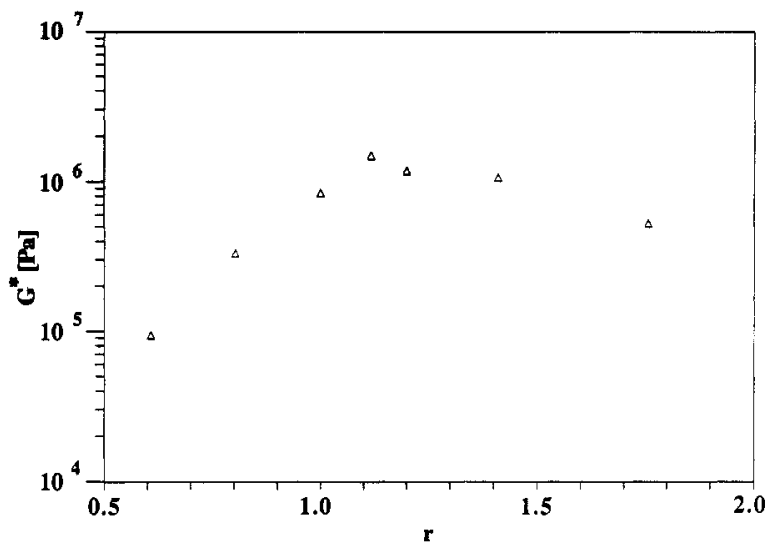

Figure 8. Complex dynamic moduli $G *$ of the fully cross-linked materials of PCL7/poly-IPDI measured at $110^{\circ} \mathrm{C}$ with shear rate of $1 \mathrm{rad} / \mathrm{s}$ plotted against stoichiometric ratio $r$.

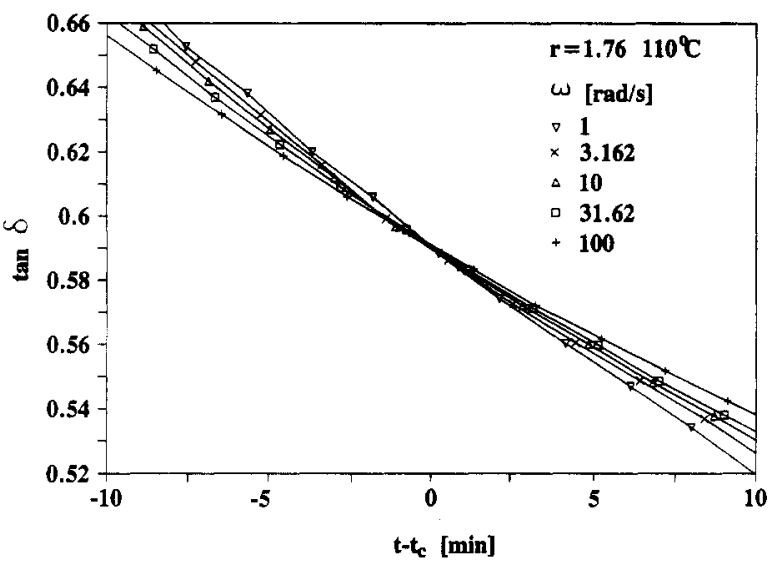

Figure 9. Evolution of $\tan \delta$ of PCL7/poly-IPDI with $r=1.76$ during isothermal cross-linking reaction at $110^{\circ} \mathrm{C}$ with the frequency of $1(\nabla), 3.162(x), 10(\Delta), 31.62(\square)$, and $100(+) \mathrm{rad} / \mathrm{s}$. The maximum strain amplitude was $10 \%$.

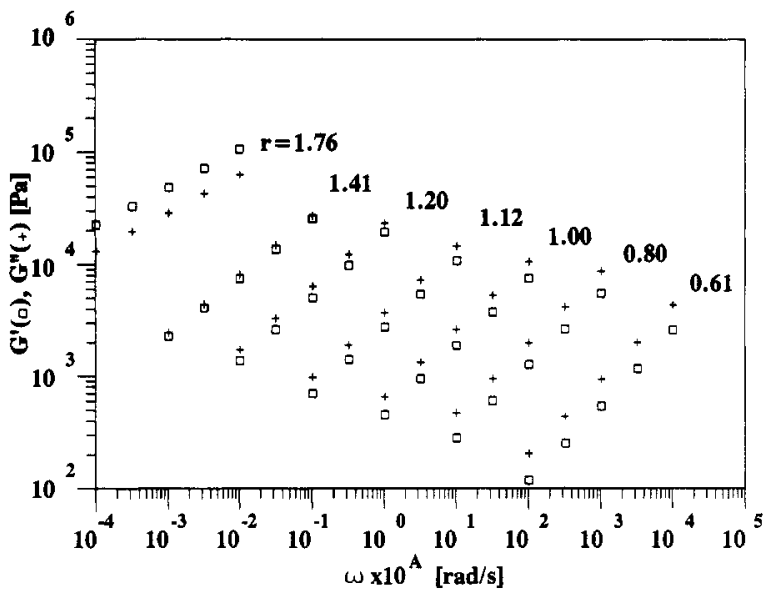

Figure 10. Dynamic storage $G^{\prime}(\square)$ and loss $G^{\prime \prime}(+)$ moduli for PCL7/poly-IPDI mixtures at the gel point plotted against frequency $\omega$. The temperature was $110^{\circ} \mathrm{C}$ and the maximum strain amplitude was $10 \%$. The curves were horizontally shifted by the factors $A=-4$ for $r=1.76,-3$ for $r=1.41,-2$ for $r=1.20$, -1 for $r=1.12,0$ for $r=1.00,1$ for $r=0.80$, and 2 for $r=0.61$, in order to avoid overlap.

The second parameter is the zero-shear viscosity $\left(\eta_{0}\right)$ of the prepolymer. Here we used the viscosity of the prepolymer, partially because we could not determine the initial viscosities of the already reacting $\mathrm{PCL} /$ poly-IPDI mixtures. This most simple choice of parameters, when used in eq 8 , already gives a close representation of the data. The dashed line in Figure 13 represents the model and squares are the data points. Discrepancies between the experimental values and the calculated values increase with decreasing $r$ (i.e., increasing $n$ ). These discrepancies would further increase if, instead of $G_{e, \max }$, the stoichiometry-dependent values of $G_{\theta}$ were used for the calculation. Although a stoichiometrically imbalanced mixture forms an imperfect network chemically, it still has potentiality to gain $G_{e, \max }$ if remaining loose ends could be connected into the network. Therefore, $G_{\theta, \max }$ is considered to be the determining material constant, which, however, depends on precursor molecular weight.

At the beginning of the cross-linking reaction, unreacted cross-linker acts as a diluent and decreases the viscosity of the mixture. Using the viscosity of the mixture (instead of that of the prepolymer) would decrease the calculated values of $S$. The magnitude of this effect depends on $n$ and concentration of cross-linker, which depend on stoichiometric ratio as well as prepolymer molecular weight. In some cases, slightly better agreement could be obtained by considering this effect. The presence of cross-linker in the fully cross-linked samples should also affect $G_{\theta}$, depending on concentration and size of the cross-linker molecule. So far, however, we could not obtain sufficient data to discuss these effects.

Molecular Weight and Stoichiometry Effect on the Relaxation Exponent. Effects of prepolymer molecular weight and stoichiometric ratio on viscoelasticity of critical gels have been reported for several systems. Scanlan et al. ${ }^{6}$ explored a PDMS system at two different prepolymer molecular weights of $1 \times 10^{4}$ and $4 \times 10^{4}$. Its entanglement molecular weight of $24500^{17}$ is between the two. As the main object of the study was to investigate the effect of stoichiometry and dilution, only two data points are available to see the effect of molecular weight on $n$ and $S$ with the same stoichiometry and dilution. However, we recognize the same tendency, namely that $n$ decreases and $S$ increases with increase of molecular weight as observed in the PCL system. For example, in the case of the materials with balanced stoichiometry and no diluent, $n$ was determined as about 0.5 and 0.2 and $S$ was $\sim 10^{3}(\mathrm{~Pa}$ $\left.\mathrm{s}^{0.5}\right)$ and $\sim 10^{4}\left(\mathrm{~Pa} \mathrm{~s}^{0.2}\right)$ for the short and the long prepolymer molecules, respectively. As regards the stoichiometric ratio effect, the relaxation exponent of the short molecule decreased from about $2 / 3$ to 0.5 with cross-linker concentration increasing to $r=1$ and then it became almost independent of $r$. This observation seemed to be the same for the two molecular weights. For the long molecule, $n$ decreased from about 0.4 to 0.2 at $r \leq 1$ and adopted a constant value of about 0.2 at $r>1$.

Chambon et al. ${ }^{18}$ cross-linked a polyurethane system consisting of monodisperse poly (propylene oxide) (PPO) with a molecular weight range of 500-2000 and reported that $n$ did not depend on $M_{\mathrm{n}}$. At balanced stoichiometry, the relaxation exponent was consistently at about 0.5 and $S$ increases with decreasing $M_{\mathrm{n}}$. They also observed a significant increase of $T_{\mathrm{g}}$ induced by cross-linking, depending on the molecular weight. It, therefore, is conceivable that the molecular weight effect on $S$ and $n$ is somewhat masked by the closeness to $T_{\mathrm{g}}$, which also depends on prepolymer molecular weight. In comparison, the increase of $T_{\mathrm{g}}$ of PCL due to cross-linking is very small even at high conversion of the cross-linking reaction as shown in Table II. Vitrification has only negligible effects on PCL rheology at the experimental temperature of 110 ${ }^{\circ} \mathrm{C}$. Therefore as far as the molecular weight dependence of $S$ and $n$ is concerned, these two results cannot be directly compared. Investigations of the stoichiometry effect ${ }^{19}$ by using a PPO with molecular weight of 965 agreed well 
Table IV

Relaxation Exponent $n$ and Gel Strength $S$ of PCL7 Critical Gels

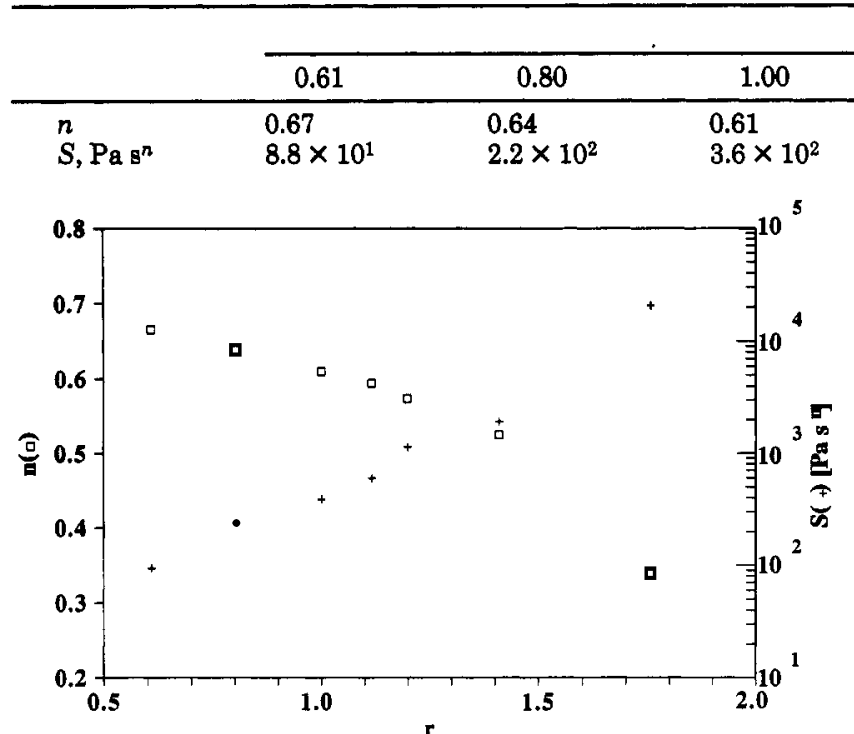

Figure 11. Relaxation exponent $n(\square)$ and gel strength $S(+)$ plotted against stoichiometric ratio $r$.

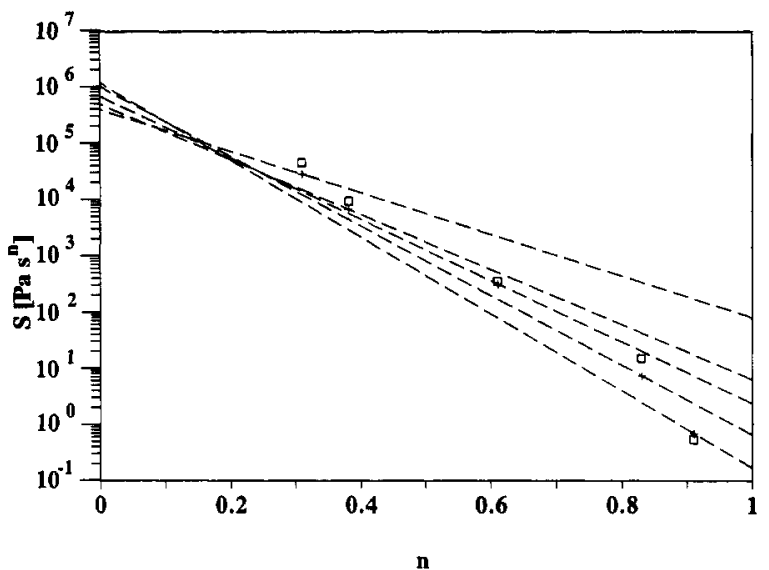

Figure 12. Experimental values ( $\square$ ) and calculated values ( + ) of the gel strength $S$ plotted against the relaxation exponent $n$. Each dashed line connected equilibrium modulus $G_{\mathrm{e}}$ and zeroshear viscosity $\eta_{0}$ of each PCL.

with those on the short PDMS molecule, although the functionalities of cross-linker were different (4 for PDMS and 3 for PPO).

Critical gels from low $-M_{\mathrm{w}}$ precursors have been reported to have high relaxation exponents. Examples are epoxides ${ }^{9}$ and polyesters ${ }^{20}$ in bulk condensation. This same behavior seems to be prevailing with the PCL of low molecular weight.

The $n$ value obtained for PCL20 (above the entanglement molecular weight) is relatively small as found in the long molecule of PDMS. Such small $n$ values were also found in physical cross-linking systems including radiationcured polyethylene ${ }^{21}$ and crystallizing polypropylene. ${ }^{22}$ In these systems, the molecular weight between physical cross-linking points is believed to be longer than the entanglement molecular weight.

As long as prepolymer molecular weights were below $M_{\mathrm{c}}, n$ was mostly reported to be 0.5 in balanced stoichiometry systems. In this case, however, 0.5 is not a universal value but would be expected only for the prepolymer with molecular weight of $\sim 8 \times 10^{3}(r=1)$ or $r \sim 1.2\left(M_{\mathrm{n}}=\right.$ $6600)$.

Durand et al. ${ }^{8}$ found that $n$ has a value close to $2 / 3$ for a polyurethane system in which the cross-linker concen-

\begin{tabular}{llll}
1.12 & 1.20 & \multicolumn{1}{c}{1.41} & \multicolumn{1}{c}{1.76} \\
\hline 0.59 & 0.57 & 0.52 & 0.34 \\
$5.6 \times 10^{2}$ & $1.1 \times 10^{3}$ & $1.8 \times 10^{3}$ & $1.9 \times 10^{4}$
\end{tabular}

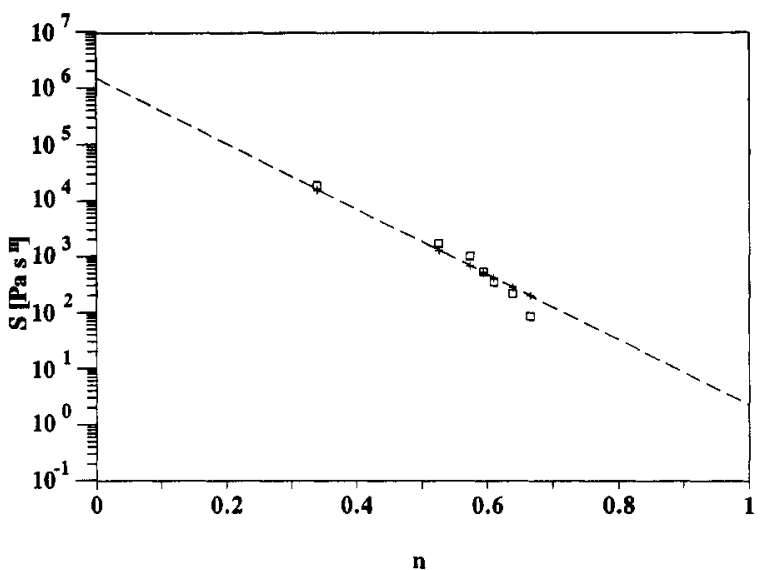

Figure 13. Experimental values ( $\square$ ) and calculated values ( + ) of the gel strength $S$ plotted against the relaxation exponent $n$. A dashed line connected equilibrium modulus $G_{\theta}$ of the fully cross-linked material of PCL7/poly-IPDI with $r=1.12$ and zeroshear viscosity $\eta_{0}$ of PCL 7 .

tration was sufficiently offset to achieve a critical gel at complete conversion. Recently, Muller et al. ${ }^{23}$ reported that the two values $(0.5$ and $2 / 3)$ could be observed in a poly(ethylene oxide) system diluted by dioxane, depending on stoichiometry. Our observation also suggests that $n$ becomes close to $2 / 3$ with $r$ approaching the lower critical limit. In the region of excess amounts of cross-linker, it may become independent of $r$ as observed in the PDMS and PPO systems. Such a lower limiting value of $n$ has not been found for the PCL system.

Experimental results seem to suggest that there is no universal value of $n$, while some theories predict a singular $n$ value for all critical gels. Theories relate the dynamic exponent $n$ with information about the molecular structure at GP. The structure may be represented by a fractal dimension $d_{f}$, which is defined by $R^{d_{f}} \sim M$, where $R$ is the radius of gyration and $M$ the mass of a molecular cluster. An experimental value of $n=2 / 3$ coincides with a theoretical prediction ${ }^{8,9}$ represented as

$$
n=d /\left(d_{\mathrm{f}}+2\right)
$$

where $d=3$ is the space dimension and the fractal dimension $d_{f}$ is determined to be 2.5 based on the percolation theory. ${ }^{24}$ In its derivation, it was assumed that Rouse modes dominate the dynamic behavior of each cluster in CG. The value of $2 / 3$ may be observed for a low cross-linker concentration system of PCL7 or a stoichiometrically balanced system with prepolymer molecular weight of about $5 \times 10^{3}$, but $2 / 3$ is not a universal value for all PCLs.

All the CGs from stoichiometrically balanced mixtures should have similar network structures. Their main differences originate from variations in the strand length between cross-linking points. Increasing strand length might enhance the excluded volume effect. To account for this, Muthukumar ${ }^{10}$ suggested that the fractal dimension, if the excluded volume effect is fully screened, becomes

$$
\bar{d}_{\mathrm{f}}=2 d_{\mathrm{f}} /\left(d+2-2 d_{\mathrm{f}}\right)
$$


In this case, eq 9 should be written as

$$
n=d\left(d+2-2 d_{\mathrm{f}}\right) / 2\left(d+2-d_{\mathrm{f}}\right)
$$

The lower limit is given by $n=0$ (resulting in $d_{\mathrm{f}}=2.5$ with $d=3$ ) and the upper limit is prescribed by the maximum fractal dimension $d_{\mathrm{f}}=3$ (resulting in $n=2 / 3$ ). Therefore, $n$ is allowed a range of values $0 \leq n \leq 2 / 3$, depending on the extent of screening. Since eqs 9 and 11 are based on the Rouse model, they might not be applicable to the system in which there are entanglements. However, only the values obtained for the CGs of PCL2 and PCL4, which supposedly are free of entanglement, are outside of the expected range.

A difference of reactivity of the functional groups of cross-linker molecules might also affect the fractal dimension. It was pointed out that, if two of the functional groups of the cross-linker have higher reactivity than the rest, chain extension reaction dominates at the initial stage of the cross-linking reaction, so that in the vicinity of GP the growth of the network structure becomes similar to "vulcanization".25 This may change the fractal dimension of CG from the value predicted for the percolation theory to the value of the branching theory. ${ }^{26,27}$ Although we may not have to consider this phenomenon as long as each mixture follows the same chemistry, the stoichiometry effects might be explained by this transition from crosslinking to vulcanization. Let us consider the case of the lowest concentration of cross-linker that can give CG ( $r$ $=0.5$ in this case). When the cross-linking reaction stops exactly at GP, due to the deficiency of the cross-linker, all functional groups of the cross-linker have reacted while there remain significant amounts of unreacted end groups of polymer chains. Consequently, this particular CG consists of only three functional cross-linking points. With increasing concentration of the cross-linker, the CG contains a larger and larger fraction of partially reacted cross-linker and unreacted cross-linker. Cross-linker molecules of which two groups have reacted act just as chain extenders in CG, and the degree of chain extension structure, which depends on the stoichiometry, should affect the fractal dimension as discussed before.

Although real systems involve these factors which affect the fractal dimension, the above equations suggest that the relaxation exponent increases with decrease of the fractal dimension. Our observation, therefore, can be qualitatively elucidated: As the prepolymer molecular weight or the stoichiometric ratio is decreased, the structure becomes more open and $d_{\mathrm{f}}$ is lower, leading to an increase in $n$ and decrease in $S$.

\section{Conclusions}

The relaxation of poly( $\epsilon$-caprolactone) critical gels follows a power law in time (CW spectrum). The critical gel rheological properties, $n$ and $S$, strongly depend on the molecular weight and the stoichiometric ratio, assuming values over the entire possible ranges. Limits are prescribed by $\eta_{0}$ and $G_{e}$ of the polymer system. An exper- imental representation of the molecular weight dependent $S$ values is based on the molecular weight dependence of the initial viscosity $\left(\eta_{0}\right)$ and of the final modulus $\left(G_{e}\right)$ of the fully cross-linked polymers: $S=G_{\mathrm{e}}\left(\eta_{0} / G_{\mathrm{e}}\right)^{n}$. The molecular weight dependence then enters through $\eta_{0}$ and $G_{\mathrm{e}}$. This equation is valid for a wide range of stoichiometric ratios.

The relaxation exponent of PCL critical gels decreases with increasing molecular weight and stoichiometric ratio. The theories cannot predict the experimental values of $n$ but suggest that the fractal dimension increases with increasing molecular weight and stoichiometric ratio.

Acknowledgment. We gratefully acknowledge Daicel Chemical Industries, Ltd., for financial support and providing well-characterized samples and DSC measurements. We also thank Huels AG for samples of the crosslinker.

\section{References and Notes}

(1) Berry, C. G.; Fox, T. G. Adv. Polym. Sci. 1968, 5, 261

(2) Colby, R. H.; Fetters, L. J.; Graessley, W. W. Macromolecules $1987,20,2226$.

(3) Treloar, L. R. G. The Physics of Rubber Elasticity; Clarendon Press: London, 1975.

(4) Chambon, F.; Winter, H. H. Polym. Bull. 1985, 13, 499. Winter, H. H.; Chambon, F. J. Rheol. 1986, 30, 367

(5) Chambon, F.; Winter, H. H. J. Rheol. 1987, 31, 683.

(6) Scanlan, J. C.; Winter, H. H. Macromolecules 1991, $24,47$.

(7) Muthukumar, M. J. Chem. Phys. 1985, 83, 3161.

(8) Durand, D.; Delsanti, M.; Adam, M.; Luck, J. M. Europhys. Lett. 1987, 3, 97.

(9) Martin, J. E.; Adolf, D.; Wilcoxon, J. P. Phys. Rev. Lett. 1988 , $61,2620$.

(10) Muthukumar, M. Macromolecules 1989, 22, 4656.

(11) Lipshitz, S. D.; Macosko, C. W. Polym. Eng. Sci. 1976, 16, 803.

(12) Lipshitz, S. D.; Macosko, C. W. J. Appl. Polym. Sci. 1977, 21, 2029.

(13) Guise, G. B.; Smith, G. C. J. Appl. Polym. Sci. 1980, 25, 149.

(14) Busfield, W. K. J. Macromol. Sci., Chem. 1982, A17, 297.

(15) Flory, P.J.Principles of Polymer Chemistry; Cornell University Press: Ithaca, NY, 1953.

(16) Leung, Y.; Eichinger, B. E. J. Chem. Phys. 1984, 80, 3885.

(17) Ferry, J. D. Viscoelastic Properties of Polymers; John Wiley and Sons: New York, 1980.

(18) Chambon, F.; Petrovic, Z. S.; MacKnight, W. J.; Winter, H. H. Macromolecules 1986, 19, 2146.

(19) Winter, H. H.; Morganelli, P.; Chambon, F. Macromolecules $1988,21,532$.

(20) Rubinstein, M.; Colby, R. H.; Gilmor, J. R. Polym. Prepr. (Am. Chem. Soc., Div. Polym. Chem.) 1989, 30, 81.

(21) Valles, E. M.; Carella, J. M.; Winter, H. H.; Baumgaertel, M. Rheol. Acta 1990, 29, 535.

(22) Lin, Y. G.; Mallin, D. T.; Chien, J. C. W.; Winter, H. H. Macromolecules 1991, 24, 47.

(23) Muller, R.; Gerard, E.; Dugand, P.; Rempp, P.; Gnanou, Y. Macromolecules 1991, 24, 1321.

(24) Stauffer, D. Introduction to Percolation Theory; Taylor \& Francis: London, 1985.

(25) de Gennes, P.-G. Scaling Concepts in Polymer Chemistry; Cornell University Press: Ithaca, NY, 1979.

(26) Stockmayer, W. H. J. Chem. Phys. 1943, 11, 45.

(27) Zimm, B.; Stockmayer, W. H. J. Chem. Phys. 1949, 17, 1301.

Registry No. IPDI (copolymer), 139582-82-4. 